\title{
Hysteretic Control Influence Operators Representing Smart Material Actuators: Identification and Approximation
}

\author{
H.T. Banks \\ Center for Research in Scientific Computation \\ Box 8205 \\ North Carolina State University \\ Raleigh, NC 27695-8205 \\ e-mail: htbanks@eos.ncsu.edu \\ and \\ A.J. K11rdila \\ Dept. of Aerospace Engineering \\ Texas A\&M University \\ College Station, TX 77843
}

\section{Introduction}

A large class of emerging actuation devices and materials exhibit strong hysteresis characteristics during their routine operation. For example, when piezoceramic acturators are operated under the influence of substantial electric fields, it is known that the resulting input-output behavior is mildly hysteretic. Tikewise, when shape memory alloys are resistively heated to induce phase transformations, the input-output response at the structural level is also known to be strongly hysteretic. This note discusses mathematical issues that arise in identifying a class of hysteresis operators that have been employed for modeling shape memory alloy actuation. Specifically, the identifiability of a class of distributed hysteresis operators that arise in the control influence operator of a class of second order evolution equations is investigated. In this paper we introduce distributed, hysteretic control influence operators derived from smoothed Preisach operators $[\mathrm{V}, \mathrm{M}]$ and generalized hysteresis operators derived from results of Krasnoselskii and Pokrovskii. For these classes, the identification problem in which we seek to characterize the hysteretic control influence. operatior can he expressed as an output least square minimization over probability measures defined on a compact subset of a closed half-plane. Consistent and convergent approximation methods for identification of the measure characterizing the hysteresis are readily obtained from our formulations. .

Research in active, or smart materials, for vibration attenuation, shape control and micro-mechanical actuation is proceeding at a rapid pace. As acturation devices based on the electro-mechanical behavior of piezoceramics and the shape memory alloys have become more widespread, it is now well-appreciated that this class of actuation devices exhibits significant nonlinear hysteretic response.

Shape memory alloys are a class of metal compounds which possess the capability to sustain and recover relatively large strains $(\approx 10 \%)$ without undergoing plastic deformation. These unique material characteristics are due in large part to the materials' ability to undergo internal crystalline transformations in the presence of external applied stress and/or changes in temperature. These transformations from the parent phase, austenite, at stress free, high temperature conditions to several variants of the low temperature martensite phase, are also a function of the history of the material. While these materials are very much in the category of emerging technologies, several of them are currently commercially available and have been used in engineering applications. Among the most popular are the nickel-titanium alloy known as Nitinol and copper zinc aluminum alloys. NiTi can be used in high performance devices with recoverable strain in the 
$6 \%$ range while $\mathrm{CuZnAl}$ performs well in very high cyclic load situations with recoverable strains of approximately $2 \%$.

The phenomenolngical responses in SMA are reasonably well understood. If one takes a stress-free SMA at high temperature in the austenite or parent phase and cools it, a gradual transformation to the low temperature or martensite phase is achieved. Several variants of the martensite including multiple twins are obtrained in this conling process.

The thermally induced phase transitions are the basis of an explanation of the strain recovery features of SMA. If the SMA is in the martensite phase and a unidirectional stress $\sigma$ is applied, at a temperatume dependent critical stress $\sigma_{\text {crit }}=\sigma_{\text {crit }}(T)$ detwinning of the martensite variants begins and eventually results in a single variant of detwinned martensite aligned with the axis of the stress loading. A similar state is achieved under the loading if one starts with an SMA in the austenite phase. During these phase transformations the internal stress in the SMA changes only slightly and a significant apparently plastic strain is achieved. If $T$ is significantly low during the stress induced martensite phase transformation, a large residual strain $\varepsilon_{r}$ remains after unloading. This strain can be recovered by heating the SMA; this is termed the "shape memory effect" or SME. This recovery can be free (no work is done), fully restrained (the SMA is restrained from recovery of its original dimension and geometry thereby producing large internal stresses), or controlled (the SMA is constrained so partial recovery of the residual strain is achieved but some stress is present to prevent full recovery).

It is this SME feature of SMA that can be exploited to develop distributed force actuators to be used as controllers in composite materials. In a typical example one employs SMA fibers reinforcing a non-SMA composite structure, e.g., SMA fibers in an elastic matrix. The SMA fibers or "wires" are stress loaded and deformed at low temperatures in the martensitic phase. They are then unloaded to generate some martensitic residual strain. Once embedded in the elastic matrix they can be heated (using an electrical or thermal input) to achieve residual strain recovery. In Nitinol embedded composite structures one can produce up to $10^{6} \mathrm{psi}$ in constrained recovery.

To help motivate the discussion of hysteresis modeling that follows in the next section, we consider the resultis of experiments measuring the structural re- sponse of a beam that undergoes deformation under the stress induced in resistively heated shape memory alloy wires. The experimental setup is rather simple. A thin aluminum beam, 1/32 inch in thickness, is catilevered at one end as depicted in Figure 1. A "two-way" shape memory alloy wire is attached to the rigid hase supporting the cantilevered heam, and to an offset attached to the tip of the free end of the beam. A thermocouple is attached in the center of the length of the shape memory alloy wire, and a strain gauge is attached on the surface of the beam, also at its midpoint. It is clear that with this simple experiment, the temperature (input) to the shape memory alloy wire and the output strain at the surface of the beam can be collected to characterize a temperature-to-strain plant model. Figure 2 depicts the results of a series of experiments wherein the current that resistively heats the wire is varied. In Figure 2a, the current in the wire is held constant at 3 amps until a target temperature is achieved, then the current is turned off. As is apparent from the experiment, the major ascent loops and descent lonps are strongly hysteretic. In Figure $2 \mathrm{~b}$, the same protocol in another experimental run is followed, except that the current during activation is 2.5 amps. Not only is hysteresis evident, by comparing the response strain-versus-temperature curves to those depicted in Figure 2a, the family of hysteresis curves is dependent on the input current.

It has been understond for quite some time that there are two fundamental approaches to mathematically characterizing the input-output behavior of complex dynamical systems. In one approach, investigators model a given system as a collection, or even continuum, of components for which ideal models can be derived from the principles of physics. In the second method, the overall qualitative hehavior of the system as a whole is observed, and some "best representative" is selected from a class of models that exhibit desired, empirically ohserved, properties. With respect to modeling hysteresis in active materials, for example, different researchers (including Tiang and Rogers [T, R], Brinson [Br], Barret [Ba] and Lagoudas [BL]) have formulated constitutive models for shape memory alloys. The body of research in [LR, Br, Ba] and [BL], regarded as a whole, falls within the first category described ahove. On the other hand, Hughes and Wen $\lceil\mathrm{HW}\rceil$ have utilized the Preisach model in a system theoretic sense to represent a static, hysteretic transducer that can be used to derive compensators for controlling shape memory alloy wires. The approach in this paper is also an example of the second, or system then- 
retic, approach to modeling the response of dynamical systems and is motivated strongly by the work of Hughes and Wen. In this paper we address several mathematical issues that arise in modeling and identification of a class of nonlinear, hysteretic control influence operators in second order evolution operators. Our formulation leads to consistent and covergent approximation schemes for the identification problem; we are currently using the resulting computational methods for numerical predictions with experimental results for a class of shape memory alloy actuated structures.

\section{The generalized smoothed Preisach-Krasnoselskii-Pokrovskii control output operator}

Tet. $\mathcal{S}=\left\{s \in R^{2} \mid s=\left(s_{1}, s_{2}\right), s_{1}<s_{2}\right\}$ be the Preisach half plane $[\mathrm{V}, \mathrm{KP}\rceil$ of threshold parameters used to parameterize the PKP kernels as follows. Define a fixed monotone continuous ridge function $r$ to be used as a smoothed relay operator and define its translates

$$
r_{s_{i}}(x)=r\left(x-s_{i}\right), s=\left(s_{1}, s_{2}\right) \in \mathcal{S}
$$

where $\mathcal{S}$ is the closure of $\mathcal{S}$. The functions $r_{s_{1}}, r_{s_{2}}$. define the envelope of admissible paths (see Figure 3) for the smooth PKP kernels which depend of the parameters $s=\left(s_{1}, s_{2}\right)$, a monotone input function $u(t)$ (or piecewise monotone input) and an initial state $\xi \in I \equiv\{-1,1\}$. The generalized PKP kernels are defined by a mapping

$$
(u, \xi) \rightarrow k_{s}(u, \xi): C\lceil 0, T\rceil \times I \rightarrow C\lceil 0, T\rceil
$$

parameterized by $s \in \mathcal{S}$. The definition can best be given by proceeding in three steps:

1. Define the action of the kernel on monotone input functions $u_{m} \in C[0, T]$

2. Define the action of the kernel on piecewise monotone input functions $u_{p m} \in C[0, T]$

3. Fxtend by density arguments the definition in (ii) for arbitrary $u \in C\lceil 0, T\rceil$.

For any monotone function $u_{m}(t) \in C[0, T]$, define the monotone output operator

$\left\lceil\mathcal{M}\left(u_{m}, \xi\right)\right](t)=\left\{\begin{array}{l}\max \left\{\xi, r\left(u_{m}(t)-s_{2}\right)\right\} \text { if } u_{m} \nearrow \\ \max \left\{\xi, r\left(u_{m}(t)-s_{1}\right)\right\} \text { if } u_{m} \searrow .\end{array}\right.$
Next suppose we are given the continuous piecewise monotone input function $u_{p m} \in S_{1, j}[0, T]$ where $S_{1, j}$ is the set of continunus piecewise linear splines with $j$ knots in $[0, T]$. The definition in this case is defined recursively on each subinterval. First, we set $\mathcal{M}_{0} \equiv \xi$ and define

$\left[k_{s}\left(u_{p m}, \xi\right)\right](t)=\left\{\begin{array}{r}{\left[\mathcal{M}\left(u_{p m}, \mathcal{M}_{k-1}\right)\right](t)} \\ t \in\left[t_{k-1}, t_{k}\right] \\ \mathcal{M}_{k}=\mathcal{M}\left(u_{p m}, \mathcal{M}_{k-1}\right)\left(t_{k}\right) \\ k=1 \ldots j .\end{array}\right.$

Obviously, for any continuous piecewise linear discretization of the input, this equation suffices for computational purposes. A typical output is depicted in Figure 4. The extension of this definition to arbitrary $u \in C\lceil 0, T\rceil$ now follows standard density arguments $[\mathrm{V}, \mathrm{KP}]$. With this definition of the hysteretic control influence operator in place we can prove for each $u \in C[0, T], \xi \in I$ and $t \in[0, T]$, the maping $s \rightarrow\left[k_{s}(u, \xi)\right](t)$ is continuous from $\mathcal{S}$ to $R^{1}$. This result plays an essential role in establishing well-posedness of the identification proceedure and the convergence of approximation schemes.

We should note that the argument $\xi \in I$ defines the initial state of the delayed relay operator, and affects the output of the delayed relay operator only if the input $u(t)$ happens to have an initial value in the open interval $\left(s_{1}, s_{2}\right)$. Tet $f \in \mathcal{B}(\mathcal{S}, l)=$ the Borel measurable functions mapping $\mathcal{S} \rightarrow I$, let $\mu \in M \equiv$ the class of finite signed Borel measures on $\mathcal{S}$, and let $u \in C[0, T]$. The generalized Preisach operator $P_{\mu}(u, f)$ is defined via

$$
\left[P_{\mu}(u, f)\right\rceil(t) \equiv \int_{\mathcal{S}}\left[k_{s}(u, f(s))\right\rceil(t) d \mu(s) .
$$

If the control influence operator $B_{\mu}$ is defined via

$$
B_{\mu}(u, f) \equiv P_{\mu}(u, f) \cdot g \equiv \int_{S}\left[k_{s}(u, f(s))\right](t) d \mu(s) \cdot g
$$

where $g \in V^{*}$ and $\mu \in M$, then

$$
B_{\mu}(u, f) \in L_{2}\left((0, T), V^{*}\right) .
$$

Here $V^{*}$ is the congugate dual of a Hilbert space $V$.

\section{Second order evolution equations}

Based on the above definitions, we present our governing equations in a weak form that incorporates the hysteretic control influence operator. Let $V$ and 
$H$ be real Hilbert spaces that form a Gelfand triple [W]. That is,

$$
V \hookrightarrow H \simeq H^{*} \hookrightarrow V^{*}
$$

Each of the embeddings $V \hookrightarrow H$ and $H^{*} \hookrightarrow V^{*}$ are dense and contimunus. The pivot space $H$ is identified with its dual space $H^{*}$ by the Riesz-map. Following [BIW, BSW], we define two parameter dependent operators $A_{1}(q)$ and $A_{0}(q)$ that represent the damping and stiffness operators, respectively. The parameters $q$ are assumed to lie in a compact metric space $\mathcal{Q}$. In operator form, the equations governing the dynamics of the system of interest to us have the form $([\mathrm{BI}])$

$$
\ddot{w}(t)+A_{1}(q) \dot{w}(t)+A_{0}(q) w(t)=\left[B_{\mu}(u, f)\right](t)
$$

in $V^{*}$.

Under standard assumptions on the operators $A_{0}, A_{1}$, we can prove (see [BIW, BSW]) that for $u \in$ $C[0, T]$ and $f \in \mathcal{B}(\mathcal{S}, I)$ and each $(q, \mu) \in \mathcal{Q} \times M$, there is a unique solution $w(q, \mu)$ such that

$$
\begin{aligned}
& w \in L_{\infty}((0, T), V) \subset L_{2}((0, T), V) \\
& \dot{w} \in L_{\infty}((0, T), H) \cap L_{2}((0, T), V) \\
& \ddot{w} \in L_{2}\left((0, T), V^{*}\right)
\end{aligned}
$$

and morenver, we actimally have

$$
\begin{aligned}
& w \in C([0, T], V) \\
& \dot{i} \quad \in C([0, T], H) .
\end{aligned}
$$

Equation (3.1) constitutes the equations governing the input/output behavior of the dynamical system. For purposes of identifying the "parameters" $(q, \mu)$ that characterize the system, we must choose a reasonable measurement, or observation, error functional. For example, the quadratic measure of output error $([\mathrm{BK}\rceil)$

$$
\begin{aligned}
. J(q, \mu)= & \int_{0}^{T}\left\{\frac{1}{2}\left|C_{1} w(q, \mu)(t)-\tilde{w}\right|_{Z_{1}}^{2}\right. \\
& \left.+\frac{1}{2}\left|C_{2} \dot{w}(q, \mu)(t)-\tilde{\dot{w}}\right|_{Z_{2}}^{2}\right\} d t
\end{aligned}
$$

is a commonly used error criterion. Here $\tilde{w}, \tilde{\dot{w}}$ denote the experimentally observed data. More generally, we may assume. I has the form

$$
J(q, \mu)=\int_{0}^{T} j(t, u(q, \mu)(t), \dot{w}(q, \mu)(t)) d \nu(t) .
$$

We are interested in minimizing this cost functional (3.2), subject to the dynamics (3.1), over $q \in \mathcal{Q}$ and $\mu \in \mathcal{A} \subset M$, where $\mathcal{A}$ is a suitable class of measures.

To specify precisely this class $\mathcal{A}$ of measures, we tiurn to results from the theory of probability measures. Let $\boldsymbol{S}_{\Delta}$ be a compact subset of $\mathcal{S}$ and let $\mathcal{A} \equiv \mathcal{P}\left(\mathcal{S}_{\Delta}\right)$ be the space of probability measures $\mu$ on $\mathcal{S}_{\Delta}$ tiaken with the Prohorov metric of convergence in distribution. As discussed in [BF, B, EK], the Prohorov metric is defined for $\mu_{1}, \mu_{2} \in \mathcal{P}\left(\mathcal{S}_{\Delta}\right)$ by

$$
\begin{gathered}
\rho\left(\mu_{1}, \mu_{2}\right) \equiv \quad \text { inf }\left\{\epsilon>0 \mid \mu_{1}(F) \leq \mu_{2}(F)^{\epsilon}+\epsilon,\right. \\
\left.F \text { closed, } F \subset \mathcal{S}_{\Delta}\right\}
\end{gathered}
$$

where $F^{\epsilon}$ denotes the usual $\epsilon$ neighborhood set for $F$. Then it is well known that $\rho\left(\mu_{k}, \mu\right) \rightarrow 0$ as $k \rightarrow \infty$ (i.e., convergence in $\rho$-metric) is equivalent to $\mu_{k}\left(F_{i}\right) \rightarrow \mu\left(F_{i}\right)$ for all Borel sets $F_{c} \subset \mathcal{S}_{\Delta}$ with $\mu(\partial E)=0$. Moreover, if we view $\mathcal{P}\left(\mathcal{S}_{\Delta}\right)$ as a subset of the dual space $C\left(\mathcal{S}_{\Delta}\right)^{*}$ where $C\left(\mathcal{S}_{\Delta}\right)$ is the usual space of continuous functions on $\mathcal{S}_{\Delta}$, convergence in the $\rho$ metric is equivalent to weak ${ }^{*}$ convergence: i.e.,

$$
\mu_{k} \rightarrow \mu \text { if and only if } \int_{\mathcal{S}_{\wedge}} h d \mu_{k} \rightarrow \int_{\mathcal{S}_{\wedge}} h d \mu
$$

for $h \in C\left(\mathcal{S}_{\Delta}\right)$.

Finally we also have that $\mathcal{A}=\mathcal{P}\left(\mathcal{S}_{\Delta}\right)$ with this metric is a compact metric space. Under suitable assumptions, the cost function $I$ of $(3.2)$ is weak ${ }^{*}$ lower semicontinous in $(q, \mu)$ on $\mathcal{Q} \times \mathcal{A}$. These results can be used to guarantee existence of solutions to the identification problem (see [BKW1] for details).

The formulation just outlined also lends itself readily to the development of computational methods and algorithms for the identification or "parameter" estimation problem involving (3.1), (3.2). Again we use results from probability theory (for a summary, see Theorem 3..3 in $[\mathrm{BF}])$. Define for $K-1,2, \ldots$, the sets

$$
\hat{\mathcal{P}}^{K} \equiv\left\{\sum_{i=1}^{K} a_{i} \delta_{s_{i}} \mid a_{i} \geq 0, \sum a_{i}=1, s_{i} \in \mathcal{R}^{K}\right\}
$$

where $\mathcal{R}^{K}$ is a set of $K$ rational pairs $s_{i}-\left(s_{i 1}, s_{i 2}\right)$ in $\mathcal{S}_{\Delta}$ such that $\bigcup_{K} \mathcal{R}^{K}$ is dense in $\mathcal{S}_{\Delta}$ and $\delta_{s_{i}}$ is the Dirac measure with atom at $s_{i}$. Then we have that $\bigcup_{K=1}^{\infty} \hat{\mathcal{P}}^{K}$ is dense in $\mathcal{P}\left(\mathcal{S}_{\Delta}\right)$ in the $\rho$ metric. This defines a sequence $\hat{\mathcal{P}}^{K}$ of finite dimensional approximating sets for $\mathcal{P}\left(\mathcal{S}_{\Delta}\right)$. In the usual manner 
(see $\lceil\mathrm{BK}, \mathrm{BSW}\rceil$ for compete details) one can also define finite dimensional approximating parameter sets $\mathcal{Q}^{M}$ (in the case that $\mathcal{Q}$ is infinite dimensional as, for example, in the case of spatially varying coefficients in concrete realizations of (3.1)) and approximating state spaces $H^{N} \subset V$. This results in a computationally tractable approximating minimization problem for (3.2) over (3.1) restricted to $H^{N}$ (i.e., Galerkin approximate systems), $\mathcal{Q}^{M}$ and $\hat{\mathcal{P}}^{K}$. One can then prove that the resulting solutions $\left(q^{N, M, K}, \mu^{N, M, K}\right)$ converge in an appropriate sense to a solution of the original problem of minimizing (3.2) over $\mathcal{Q} \times \mathcal{A}$ subject to the system (3.1) Details can be found in [BKW2]. Our initial computational efforts based on these ideas, also reported in [BKW2], are most promising.

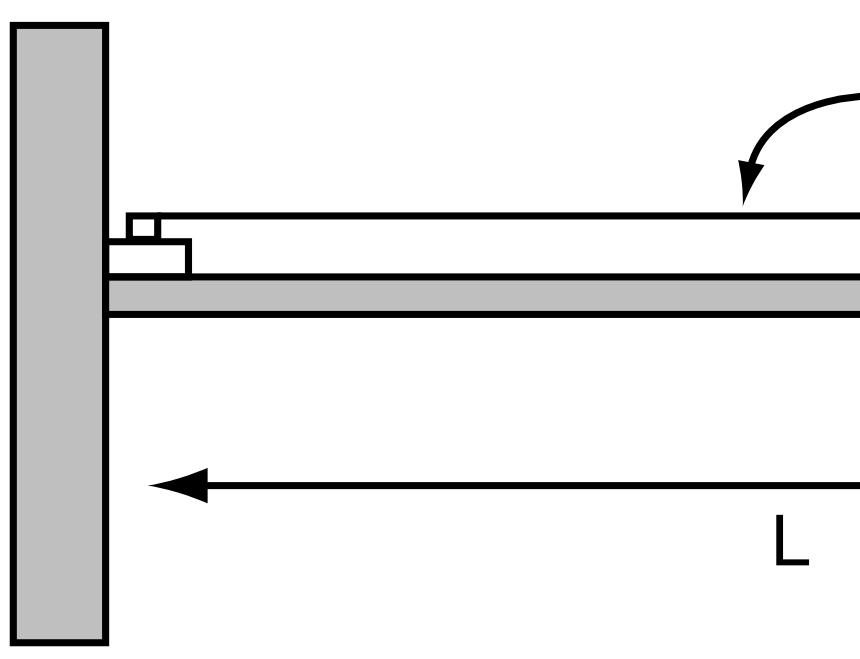

Figure 1: Experimental Setup

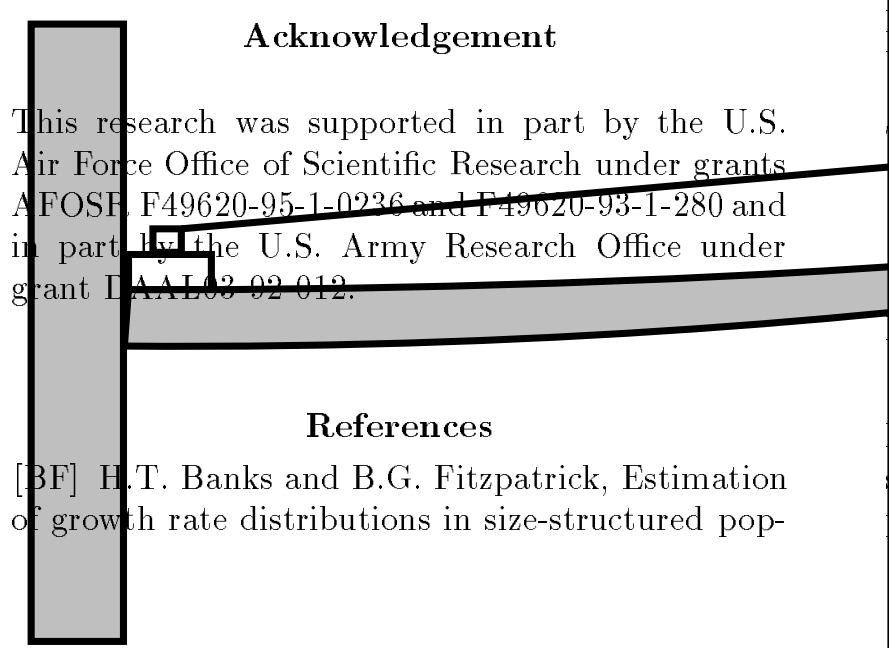

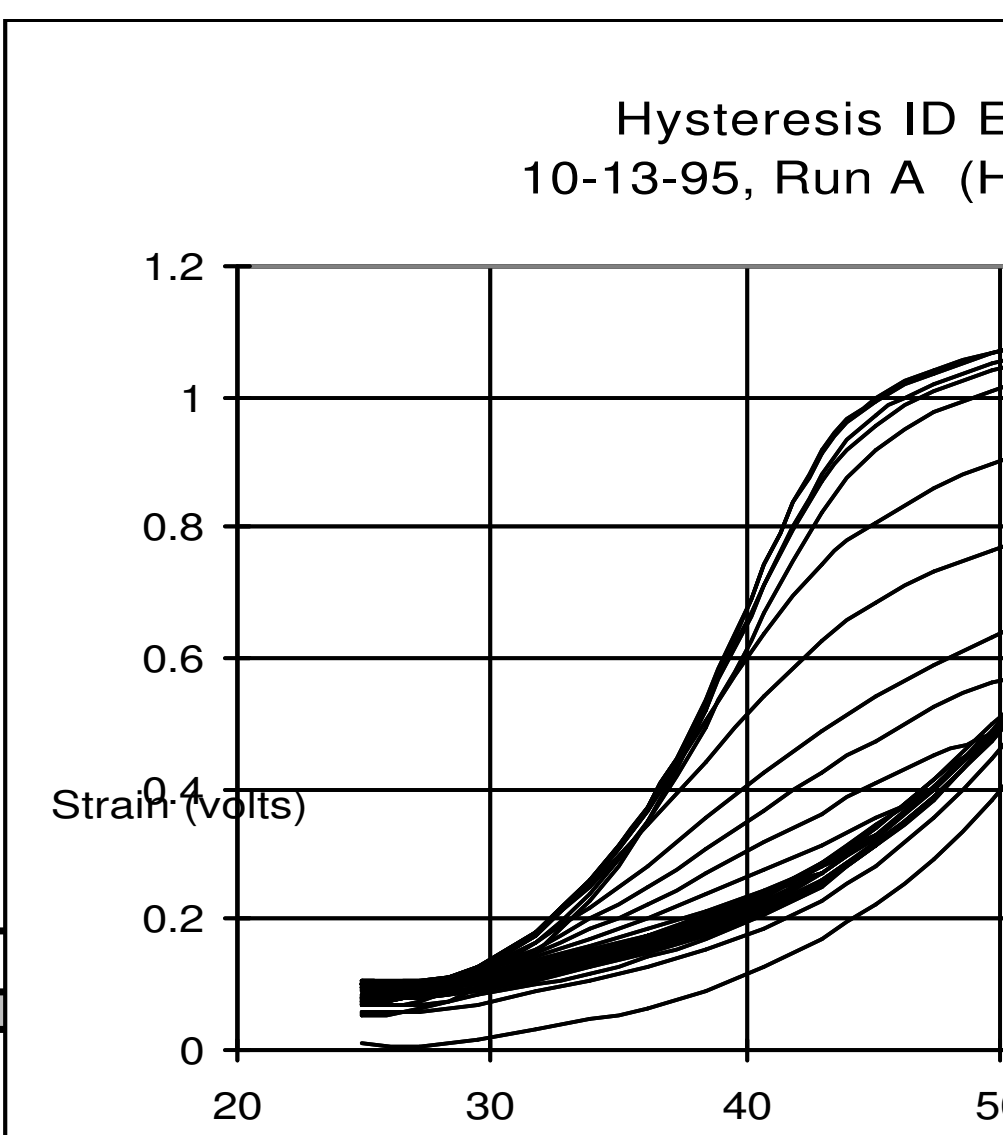

Figure 2: Hysteresis in SMA Experiments (upper) 3 Amps, (lower) 2.5 Amps

ulation models, Quart. Appl. Math. 49 (1991) 215225

[RT] H.T. Ranks and K. Tto, A unified framework for approximation in inverse problehtsisteresis ID E

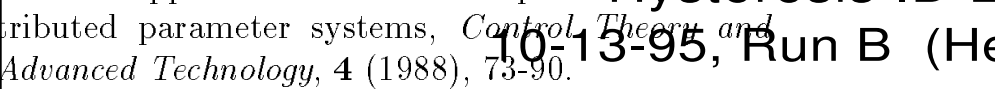

[RIW] H.T.2Ranks, K. Tin, anj Y. Wang, We tposedness for damped second drder systems with inbounded input operators, Technical Report, Center for Resedrot CPster TR93-10; Differential and Int gral Equations, 8 (1995), 587-606

BK] H.T. \$. Banks and K. Kunisch, Estimatipn Techniques for Distributed Pdrameter Systen.s. Birkhauser, Boston, 1989.

BKW11 H.T. \$anks, A.J. Kurdila, and G. W dentification of hysteretic infe-ence operato mart actudors Part I: Formulation. Technic

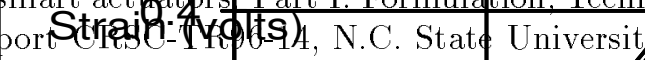

0.2 
[EK] S.N. Either and T.G. Kurtz, Markov Processes: Characterization and Convergence, Wiley, $\mathrm{r}(\mathrm{X})$ New York, 1986.

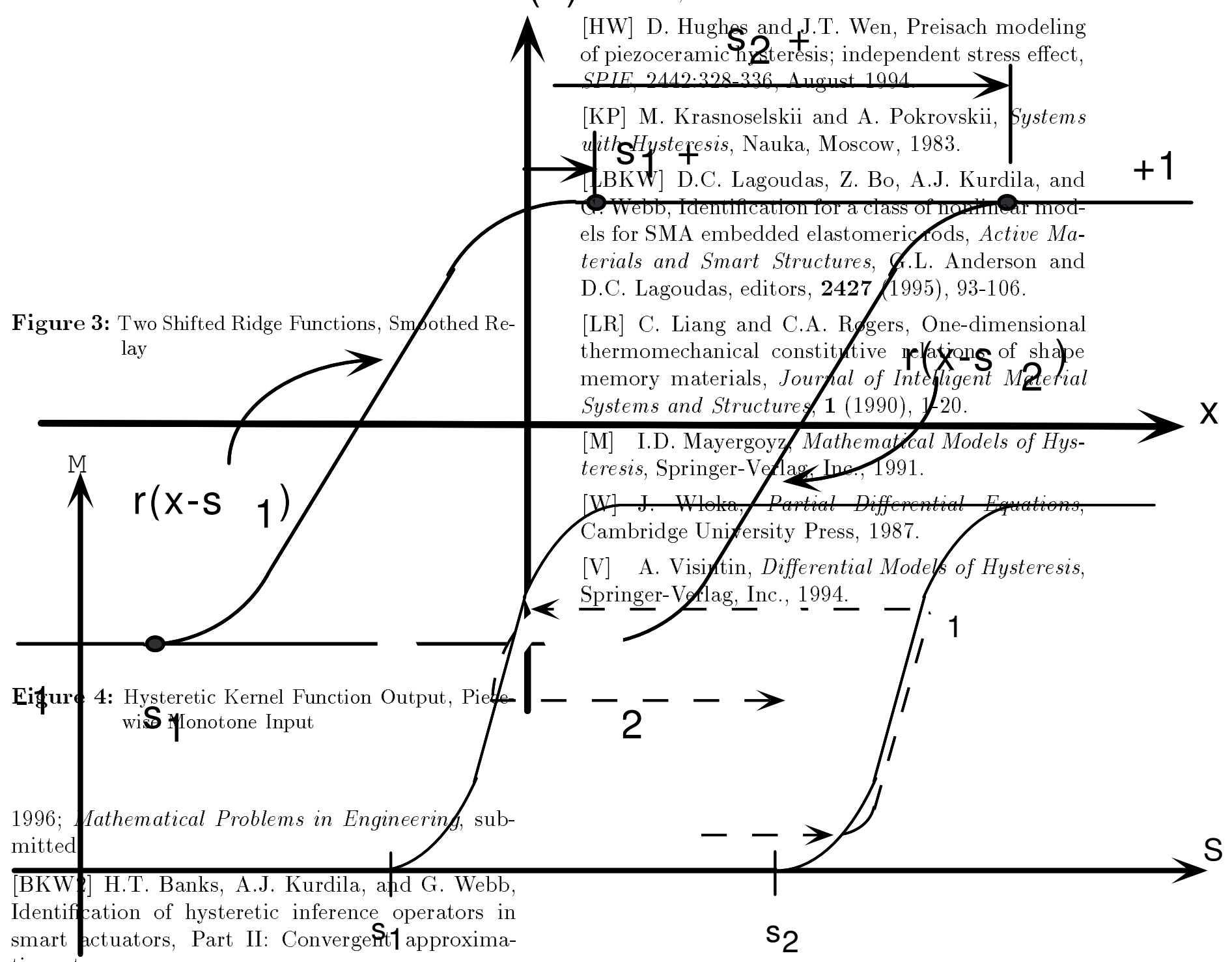

tions, to appear.

[BSW] H.T. Banks, R.C. Smith, and Y. Wang, Smart Material Strustures: Modeling, Fistimation and Control, Masson/J. Wiley, Paris/Chichester, 1996.

[Ba] D. Barret, Thermomechanical constitutive laws for shape memory alloys, preprint, 19.94.

[B] P. Billingsley, Convergense of Probobility Measures, Wiley, New York, 1968.

[BL] J. Boyd and D. Lagoudas, Thermodynamical models for constitutive laws of shape memory alloys, preprint, 1995.

[Br] C. Brinson, Constitutive laws for control of shape memory alloys, preprint, 1995. 